\title{
Cell Type Diversity in Hepatitis B Virus RNA Splicing and Its Regulation
}

\author{
Noriomi Ito, Kenji Nakashima, Suofeng Sun, Masahiko Ito and Tetsuro Suzuki* \\ Department of Virology and Parasitology, Hamamatsu University School of Medicine, Shizuoka, Japan
}

Although RNA splicing of hepatitis B virus (HBV) is a commonly observed in livers of hepatitis B patients as well as in the cultured cells replicating the viral genome, its biological significance in the HBV life cycle and the detailed regulatory mechanisms are still largely unclear. In this study, we found cell-type dependency of HBV splicing of the $3.5 \mathrm{~kb}$ pregenomic RNA, which is efficiently spliced in human hepatoma cells but not in cells derived from human hepatic stellate, mouse hepatoma and human nonhepatic cells. It may be likely that RNA splicing is one of the determinants of host range restriction of HBV. Given the finding indicating the difference in cell-type dependency of the splicing efficiency between HBV and simian virus 40, we carried out intronswapping experiments. The results suggest the presence of putative exonic splicing enhancer that possibly works in the cell-type dependent fashion. Together with further

OPEN ACCESS

Edited by: Hirofumi Akari,

Kyoto University, Japan

Reviewed by:

Kohji Moriishi,

University of Yamanashi, Japan Patrick Soussan

Université Pierre et Marie Curie,

France

${ }^{*}$ Correspondence: Tetsuro Suzuki

tesuzuki@hama-med.ac.jp

Specialty section:

This article was submitted to

Virology,

a section of the journal

Frontiers in Microbiology

Received: 23 November 2018

Accepted: 24 January 2019

Published: 08 February 2019

Citation:

Ito $N$, Nakashima $K$, Sun S, Ito $M$ and Suzuki T (2019) Cell Type Diversity in Hepatitis B Virus RNA

Splicing and Its Regulation.

Front. Microbiol. 10:207.

doi: 10.3389/fmicb.2019.00207 mutational analyses, a novel 50-nt intronic splicing silencer, whose secondary structure is well conserved among the HBV strains, was identified. It appears that this intronic silencer functions effectively independent of cell backgrounds.

Keywords: hepatitis B virus, alternative splicing, cis-acting element, intronic silencer, exonic enhancer

\section{INTRODUCTION}

Hepatitis B virus (HBV) infection is a major global problem despite the availability of an efficacious vaccine. In chronic HBV infection, liver cirrhosis and hepatocellular carcinoma (HCC) are associated with considerable morbidity and mortality. Approximately 240 million people worldwide are chronically infected with the virus (El-Serag, 2012). HBV is a member of the Hepadnaviridae family and contains a $3.2 \mathrm{~kb}$ partially double-stranded relaxed circular DNA genome with four open reading frames encoding seven proteins.

Upon infection, the uncoated viral genome is transported to the nucleus and converted into covalently closed circular DNA, which is a stable form of the viral genome and serves as the template for synthesis of viral transcripts. Four unspliced viral RNAs, 3.5, 2.4, 2.1, and $0.7 \mathrm{~kb}$, are transcribed from their respective promoters and two enhancer regions and end at common polyadenylation signal located in the core open reading frame. The $3.5 \mathrm{~kb}$ RNA includes precore and pregenomic RNA species. Precore mRNA codes for precore antigen or HBeAg. The pregenomic RNA serves as a template for the synthesis of HBV DNA and also as the mRNA of core antigen ( $\mathrm{HBcAg})$ and polymerase. In addition, the $3.5 \mathrm{~kb}$ RNA can be alternatively spliced to generate at least 14 splice variants that have been identified in sera and livers of hepatitis B patients (Candotti and Allain, 2017). In cultured human hepatoma cells transfected with the viral genome, synthesis of multiple spliced RNAs derived from $3.5 \mathrm{~kb}$ RNA has been commonly observed among HBV isolates. It has been reported that up to $80 \%$ of intracellular capsids contain the viral DNAs originated from the spliced RNAs in HBV genome-replicating hepatoma cells (Terre et al., 1991; Rosmorduc et al., 1995; 
Soussan et al., 2008; Redelsperger et al., 2012; Bayliss et al., 2013). The major spliced variant termed as SP1, which has an intron between nt 2448 and 488, may account for up to $30 \%$ of total 3.5 kb RNA (Gunther et al., 1997; Sommer et al., 2000; Duriez et al., 2017).

RNA splicing is an essential step for eukaryotic gene expression and is tightly regulated in different tissues and developmental stages. While this process depends on recognition of short well-conserved splice site sequences at the exon-intron boundaries, additional cis-acting splicing regulatory elements, which act as either enhancers or silencers of splicing and are potentially present either in exons or introns, play key roles in defining specificity and efficiency of splicing. In terms of splicing regulatory elements of $\mathrm{HBV}$, the post-transcriptional regulatory element (PRE), a highly structured cis-acting sequence of approximately $0.5 \mathrm{~kb}$, was identified within the viral transcripts. PRE is located near the $3^{\prime}$ end region of all HBV mRNAs and is known to be involved in regulation of the $3.5 \mathrm{~kb}$ RNA splicing (Heise et al., 2006). Heterologous minigene contexts were used for investigating cis-acting regulatory elements and distinct sequences that can function as positive and negative regulators for the $3.5 \mathrm{~kb}$ RNA splicing were identified within the PRE (Huang et al., 2011). HBV intronic cis-element was also examined and an intronic splicing silencer-long, ISS $_{\mathrm{L}}$, was identified (Chowdhury et al., 2011). However, biological significance of HBV RNA splicing, especially in terms of viral replication and/or host range as well as the detailed regulatory mechanisms underlying post-transcriptional processing events in the HBV life cycle are still largely unclear.

In this study, we compared expression of HBV $3.5 \mathrm{~kb}$ RNA and spliced form(s) derived from the RNA in 13 kinds of cell lines transfected with the viral genome and found that the 3.5 kb RNA can be relatively efficiently spliced in human HCC cells compared to other types of cells. Findings obtained from intronswapping experiments between HBV and simian virus 40 (SV40) suggest involvement of a putative exonic enhancer in regulation of alternative splicing in a cell-type dependent manner. Further, we identified a novel intronic silencer element spanning at nt 2877-2926 that was well conserved in the predicted structural conformation among HBV strains.

\section{MATERIALS AND METHODS}

\section{Plasmids}

Plasmids containing the 1.24-fold HBV genomes derived from HBV genotypes (GTs) A (NCBI No. AB246337), B (NCBI No. AB246342) and D (NCBI No. AB246347); pUC-HBV-Ae, -Bj56 and -D_Ind60, respectively, were gifts from Dr. Mizokami of National Center for Global Health and Medicine, Japan (Sugauchi et al., 2004a,b). pUC-HBV-Ce was 1.24-fold HBV genome derived from a consensus sequence of HBV GT-C (subgenotype Ce), as described (Sun et al., 2017). A series of deletion mutants with or without substitutions in the HBV sequence corresponding to major intron of the splicing, as indicated in Figure $\mathbf{3 A}$, were generated based on pUC HBV-Ce, -Ae and -D_Ind60, which were used as templates for PCR. pCAG-SV40 that expresses
SV40 late gene under the CAG promoter (Nakanishi et al., 2008) was provided by Dr. Nakanishi of National Institute for Longevity Sciences, Japan. HBV-based chimeric plasmid carrying the SV40 intron region, $\mathrm{pHBV} /$ int-SV40, was constructed as follows. HBV cDNA corresponding to the major splicing intron region $(1,256 \mathrm{bp})$ in $\mathrm{pUC} \mathrm{HBV}-\mathrm{Ce}$ was deleted by $\mathrm{PCR}$ and used for cloning the $932 \mathrm{bp}$ of SV40 cDNA fragment of the splicing intron region that was amplified by PCR using pCAGSV40 as a template, by using an In-Fusion HD cloning kit (Takara Bio, Shiga, Japan). In the same way, to create SV40-based chimeric plasmid carrying the HBV intron region, pSV40/int$\mathrm{HBV}$, a 1,256 bp fragment of HBV cDNA of the splicing intron was amplified, followed by cloning the fragment into the pCAGSV40-based linearized vector in which the sequence of the SV40 splicing intron was deleted. A series of mutants pUC HBV-Ce-D1, -D2, -D3, -D4, -D5, -S1/D1, -S1/D1/S2 and -S1, which carry partial deletions and/or substitutions within the region corresponding to the major intron, were generated via several PCRs using pUC HBV-Ce as the template, followed by self-ligation of the synthesized fragments. Nucleotide sequences of the regions introducing substitution mutations S1 and S2 (Figure 3A) in pUC HBV-Ce-S1/D1, -S1/D1/S2 and -S1 were shown (Supplementary Table S1). To generate a series of HBV GT-C-based chimeric plasmids harboring parts of GT-A sequences (HBV Ce/Ae1-Ce/Ae7), various parts in the HBV genome were removed from $\mathrm{pUC}-\mathrm{HBV}-\mathrm{Ce}$, followed by replacing with corresponding parts from pUC-HBV-Ae. All mutated or chimeric constructs generated were sequenced to ensure that no mutations were introduced.

\section{Cell Culture and Transfection}

Human HCC cells; HuH-7, HepG2, PLC/PRF5, FLC7 (Homma et al., 1990) and ORL8c (Kato et al., 2009), human hepatic stellate TWNT4 JP7 cell (Shibata et al., 2003; Chida et al., 2017), mouse hepatoma Hepa1c1c7 cell and human non-hepatic cells; A549 (lung carcinoma), HEK293 (embryonic kidney), HEK293T (embryonic kidney), Caco2 (colorectal adenocarcinoma), C33A (cervical carcinoma), and HeLa (cervical adenocarcinoma) were maintained in Dulbecco's modified Eagle medium supplemented with $10 \%$ fetal bovine serum. Cells were transiently transfected with plasmid DNAs using transfection reagents as follows; Lipofectamine LTX (Thermo Fisher Scientific, Waltham, MA, United States) used for HuH-7, FLC7, A549, Caco2, HeLa, Hepalc1c7, and TWNT4 JP7, Lipofectamine 3000 (Thermo Fisher Scientific) for HepG2, PLC/PRF/5, ORL8c, and C33A, and linear polyethyleneimine, MW 25,000 (Thermo Fisher Scientific) for HEK293 and HEK293T.

\section{Northern Blot and Western Blot Analyses}

Northern blotting was performed as previously described (Sun et al., 2017). Briefly, total RNA samples extracted from cells transfected with HBV plasmids were separated on $1.2 \%$ agarose gel with $7 \%$ formaldehyde. The samples were transferred to a nylon membrane (Roche Diagnostics, Basel, Switzerland), followed by cross-linking to the membrane by ultraviolet light. The blot was prehybridized with DIG Easy Hybridization buffer (Roche Diagnostics) and hybridized with an HBV RNA probe 
(Supplementary Figure S1A) labeled with DIG-11-UTP using the DIG Northern Starter Kit (Roche Diagnostics). To generate a DIG-labeled RNA probe that specifically binds to HBV 3.5 $\mathrm{kb}$ RNA and its spliced forms, a PCR fragment covering the nt 1998-2447 region was used as templates for in vitro transcription. The signals were detected with CDP-Star reagent (GE Healthcare, Buckinghamshire, United Kingdom). Western blotting was performed as previously described (Li et al., 2016). Briefly, the proteins in cell lysates were separated by SDS-PAGE and transferred onto polyvinylidene difluoride membranes. After blocking, membranes were probed with primary antibodies, followed by incubation with peroxidase-conjugated secondary antibody. Antigen-antibody complexes were visualized using ECL Prime Western Blotting Detection Reagent (GE Healthcare).

\section{Determination of Quantity Ratio of the Spliced RNA to Unspliced $3.5 \mathrm{~kb}$ RNA or Total 3.5 kb RNA Derived Species}

The ratio of the spliced HBV RNAs to unspliced 3.5-kb RNA or total (spliced and unspliced) RNAs derived from $3.5 \mathrm{~kb}$ RNA was determined in two ways; based on quantitative- (q) and semi-quantitative RT-PCRs. In experiments with the 1.24-fold full-length HBV genomes but not with their deletion mutants as shown in Figures 1C, 5 and Supplementary Figures S5, S6, spliced forms and total (unspliced plus spliced) RNAs derived from $3.5 \mathrm{~kb}$ RNA were separately determined by qRTPCR as described previously (Sun et al., 2017). In brief, total RNAs were extracted from transfected cells with TRI Reagent (Molecular Research Center, Cincinnati, OH, United States). After treatment with inhibitors for DNase I and RNase, cDNA templates were synthesized and were quantified by qPCR using the SYBR qPCR Mix kit (Toyobo, Osaka, Japan) with the primer sets, unSpF; $5^{\prime}$-TCCCTCGCCTCGCAGACG- $3^{\prime}$ and unSpR; 5'-GTTTCCCACCTTATGAGTC-3' for unspliced $3.5 \mathrm{~kb}$ RNA, and SpF; 5'-CCGCGTCGCAGAAGATCT-3' and SpR; $5^{\prime}$ CTGAGGCCCACTCCCATAGG-3' for $3.5 \mathrm{~kb}$-derived spliced RNAs. Data obtained were used for calculating the ratio of the spliced RNAs to total (unspliced plus spliced) RNAs derived from 3.5-kb RNA.

In experiments containing both with wild-type and mutated HBV genomes as shown in Figures 3, 4, semi-quantitative RTPCR was used because of difficulty in designing primers/probe for qRT-PCR that allows proper quantification of $\mathrm{HBV}$ RNA isoforms generated from a series of deletion mutants. cDNA templates were prepared as described above and $\mathrm{HBV}$ cDNAs derived from spliced- and unspliced $3.5 \mathrm{~kb}$ RNAs were amplified by PCR with the primer sets, pgRNAF; $5^{\prime}$ AGCCTCCAAGCTGTGCCTTGGGTG-3' and pgRNAR; 5'AACCACTGAACAAATGGCACTAGTAAACTGAGC-3'. PCR products were visualized by agarose gel electrophoresis. Image intensities corresponding to each spliced form as well as an unspliced form were quantified using the image processing program ImageJ (Schneider et al., 2012), followed by calculating the quantity ratio of the spliced RNAs to the unspliced RNA. A schematic diagram of positions of PCR primers used is indicated (Supplementary Figure S1A).

\section{Statistical Analysis}

The significant statistical difference of HBV pgRNA splicing ratio between cell-types was determined by Wilcoxon rank sum test. For other parametric measures, significance between two groups was determined using Student's $t$-test, while significance between four or more groups was determined using one-way ANOVA with Dunnett's test. $p$-values $<0.05$ were considered statistically significant.

\section{RESULTS}

\section{Cell-Type Dependency of HBV 3.5 kb RNA Splicing}

We first compared expression of HBV $3.5 \mathrm{~kb}$, pregenomic plus precore RNA and spliced form(s) derived from the 3.5 $\mathrm{kb}$ RNA in human liver-derived and non-liver-derived cells that were cultured for 2 days after transfection with the 1.24fold HBV genome. Northern blotting with pregenome/precore probe showed that two bands corresponding to unspliced 3.5 $\mathrm{kb}$ RNA and $3.5 \mathrm{~kb}$ RNA -derived, $2.2 \mathrm{~kb}$ spliced RNA (SP1) lacking $\sim 1.2 \mathrm{~kb}$ intron, respectively, were more abundant in HepG2 cells compared to HEK293 cells (Figure 1A). Higher expression of $3.5 \mathrm{~kb}$ RNA and HBcAg in HepG2 compared to those in HEK293 cells were confirmed by qRT-PCR (Supplementary Figure S1B) and Western blotting (Figure 1B), respectively. It is noted that HepG2 and HEK293 cells exhibited similar transfection efficiency as monitoring expression of fluorescencent protein in the transfected cells (Supplementary Figure S1C). From quantification of the band intensity in northern blotting data, the quantity ratio of SP1/unspliced $3.5 \mathrm{~kb}$ RNAs appears to be higher in HepG2 cells than that in HEK293 cells (Figure 1A).

To analyze possible cell-type dependency of HBV $3.5 \mathrm{~kb}$ RNA splicing, a panel of cell lines derived from human and mouse hepatic- as well as human non-hepatic tissues was used for expression of the HBV genome derived from GT-A, -B, and -C. Both quantitative (Figure 1C) and semiquantitative (Figure 1D) RT-PCR analyses showed variation of the splicing efficiency among cell lines tested. It is of interest that the ratio of spliced/unspliced $3.5 \mathrm{~kb}$ RNAs was higher in all five kinds of cell lines derived from human HCC (HepG2, HuH-7, PLC/PRF/5, FLC7, and ORL8c), compared to other cell types; mouse hepatoma (Hepalclc7), human hepatic stellate cell derived (TWNT4), human non-hepatic cells (A549, HEK293, HEK293T, Caco2, C33A and HeLa). To ask whether such a cell-type dependent manner of the splicing was also observed in the splicing of another viral RNA, a construct carrying the SV40 late gene (SV40-L WT) was introduced into HepG2, HuH-7, PLC/PRF/5, A549, HeLa and HEK293 cells and the RNA expression was analyzed by RT-PCR. Spliced RNAs including the dominant form that corresponds to the SV40 VP1 RNA were similarly detectable both in hepatic and non-hepatic cells (Supplementary Figure S3; upper). It is thus likely that the splicing efficiency of HBV $3.5 \mathrm{~kb}$ RNA is dependent on cellular context and the RNA 


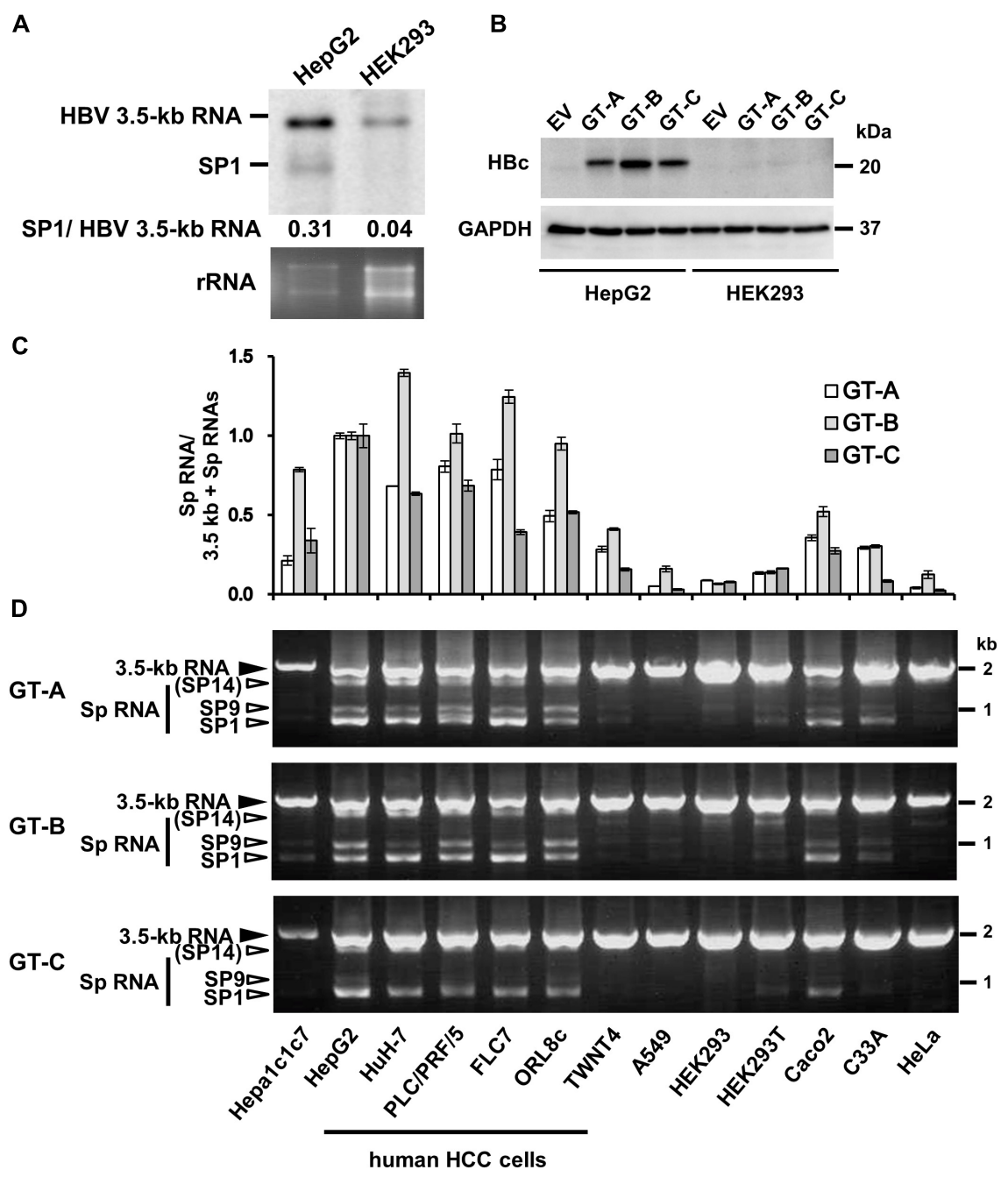

FIGURE 1 | Cell-type dependency of HBV 3.5 kb RNA splicing. (A) pUC-HB-Ce was transfected into HepG2 and HEK293 cells. Two days after the transfection, total RNA was extracted from cells and separated on an agarose gel. HBV $3.5 \mathrm{~kb}$ RNA and spliced RNA(s) were detected by northern blotting using HBV RNA probe (nt 1998-2447) (Supplementary Figure S1A). Band intensities of $3.5 \mathrm{~kb}$ - and SP1 RNAs on the blot were determined by ImageJ software and the ratios of SP1 RNA to $3.5 \mathrm{~kb}$ RNA calculated were indicated. Ethidium bromide-stained agarose gel electrophoresis of 18S ribosomal RNA (rRNA) was also shown (bottom). (B) HepG2 and HEK293 cells transfected with pUC-HB-Ae (GT-A), -Bj56 (GT-B), -Ce (GT-C) and an empty vector (EV) were analyzed by immunoblotting to detect $\mathrm{HBcAg}$ and glyceraldehyde-3-phosphate dehydrogenase (GAPDH). (C) qRT-PCR analysis was performed to determine the levels of $3.5 \mathrm{~kb}$ RNA and spliced RNA in 13 kinds of cells transfected with pUC-HB-Ae (GT-A), -Bj56 (GT-B), and -Ce (GT-C). The quantity ratios of the spliced RNAs to total 3.5 kb RNA derived RNA species were calculated and those in HepG2 cells were set to 1. Values shown represent means \pm SD obtained from three independent samples. (D) Total RNAs obtained from the cell samples as shown in (C) were used with semi-quantitative RT-PCR. cDNA bands corresponding to unspliced $3.5 \mathrm{~kb}$ RNA and its spliced forms (Sp RNA) were detected by agarose gel electrophoresis. Unspliced 3.5 kb RNA, SP9, and SP1 were identified by nucleotide sequencing. SP14 was estimated from the size of PCR product obtained. Cell lines used were indicated at the bottom.

is preferentially spliced in cells derived from human liver; in particular, HCC.

\section{Identification of Cis-Regulatory Elements Involved in HBV 3.5 kb RNA Splicing in Cell-Type Dependent and - Independent Manners}

To identify regulatory cis-element(s) involved in controlling HBV $3.5 \mathrm{~kb}$ RNA splicing in the cell-type dependent manner, two swapped-region constructs (HBV/int-SV40 and SV40/int-HBV) in which the HBV sequence corresponding to the intron for majority of the HBV spliced RNAs and the SV40 sequence of the intron for the viral late gene were swapped in the plasmids that contain the SV40 late gene (SV40-L WT) and the1.24-fold HBV genome derived from GT-C (HBV WT), respectively, were generated (Figure 2A) and the splicing patterns expressed from the swapped constructs were tested in HepG2 and HEK293 cells (Figures 2B,C). In HepG2 cells expressing HBV/int-SV40, two spliced RNAs termed HS3 and HS4 but not unspliced RNA were observed. In HEK293 cells, two spliced RNAs; HS2 and HS4 in addition to unspliced RNA (HS1) were detectable (Figure 2B). 

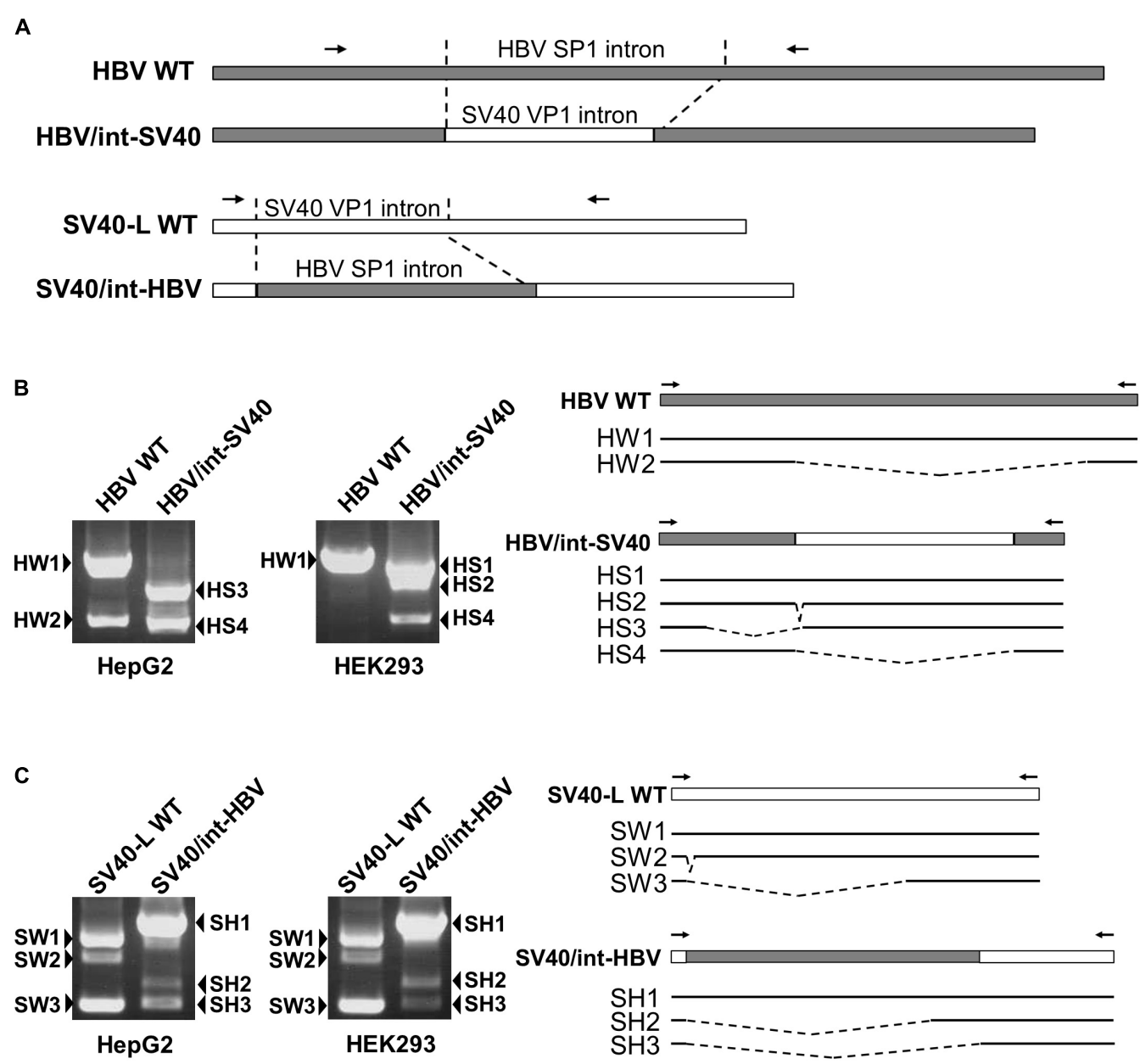

FIGURE 2 | Identification of cis-regulatory elements involved in HBV 3.5-kb RNA splicing in cell-type dependent and -independent manners. (A) A schematic representation of constructs and PCR primers used in the intron-swapping experiment is indicated. (B) HBV WT and HBV/int-SV40 were expressed in HepG2 and HEK293 cells. PCR products were analyzed by agarose gel electrophoresis (left). Schematic of unspliced and spliced forms detected is shown (right). (C) SV40-L WT and SV4O/int-HBV were expressed in the cells and PCR products were analyzed as shown in (B).

By contrast, the RNA pattern expressed from SV40/int-HBV was comparable in HepG2 and HEK293 cells. In both cells, the ratio of spliced/unspliced RNAs appeared to be lower in case of SV40/intHBV compared to SV40-L WT (Figure 2C), suggesting the existence of splicing silencer(s) in the intron region of HBV 3.5 $\mathrm{kb}$ RNA which presumably functions in the cell-type independent manner. To confirm this, SV40/int-HBV was further expressed in HuH-7, PLC/PRF/5, A549 and HeLa as well as HepG2 and HEK293 cells. As expected, expressed RNAs from SV40/int-HBV were similarly processed in all cell lines tested in contrast to the RNAs from HBV/int-SV40 and their splicing efficiency was lower compared to that from SV40-L WT (Supplementary Figure S3; upper, middle and lower).

Regarding the role of $\mathrm{HBV}$ exonic region in the splicing regulation, while a certain level of unspliced SV40 RNA in addition to the spliced forms was detectable in SV40-L WT-expressing HepG2 cells (Figure 2C), most of all RNAs expressed from HBV/int-SV40 (Figure 2B) were spliced in the cells. By contrast, higher splicing efficiency caused by
HBV/int-SV40 was not observed in HEK293 cells; it appears that the ratio of spliced/unspliced RNAs was lower in the cells expressing HBV/int-SV40 (Figure 2B) compared to SV40-L WT (Figure 2C). Such a difference in splicing efficiency which is higher in $\mathrm{HBV}$ /int-SV40 expression compared to SV40-L WT was also observed in HuH-7 and PLC/PRF/5 but not in A549 and HeLa cells (Supplementary Figure S3; upper, middle, and lower). It is thus likely that the sequence(s) within the exon region of majority of the HBV spliced RNAs plays a positive role in its splicing regulation in the cell-type dependent manner.

\section{Characterization of the Intronic Sequence Important for Silencing of the 3.5-kb RNA Splicing Within the Viral Major Intron Region}

A previous study has identified an $\mathrm{ISS}_{\mathrm{L}}$ for $\mathrm{HBV}$ pregenomic RNA splicing in expression of $\mathrm{HBV}$ minigene constructs under the control of heterologous CMV-IE promoter 


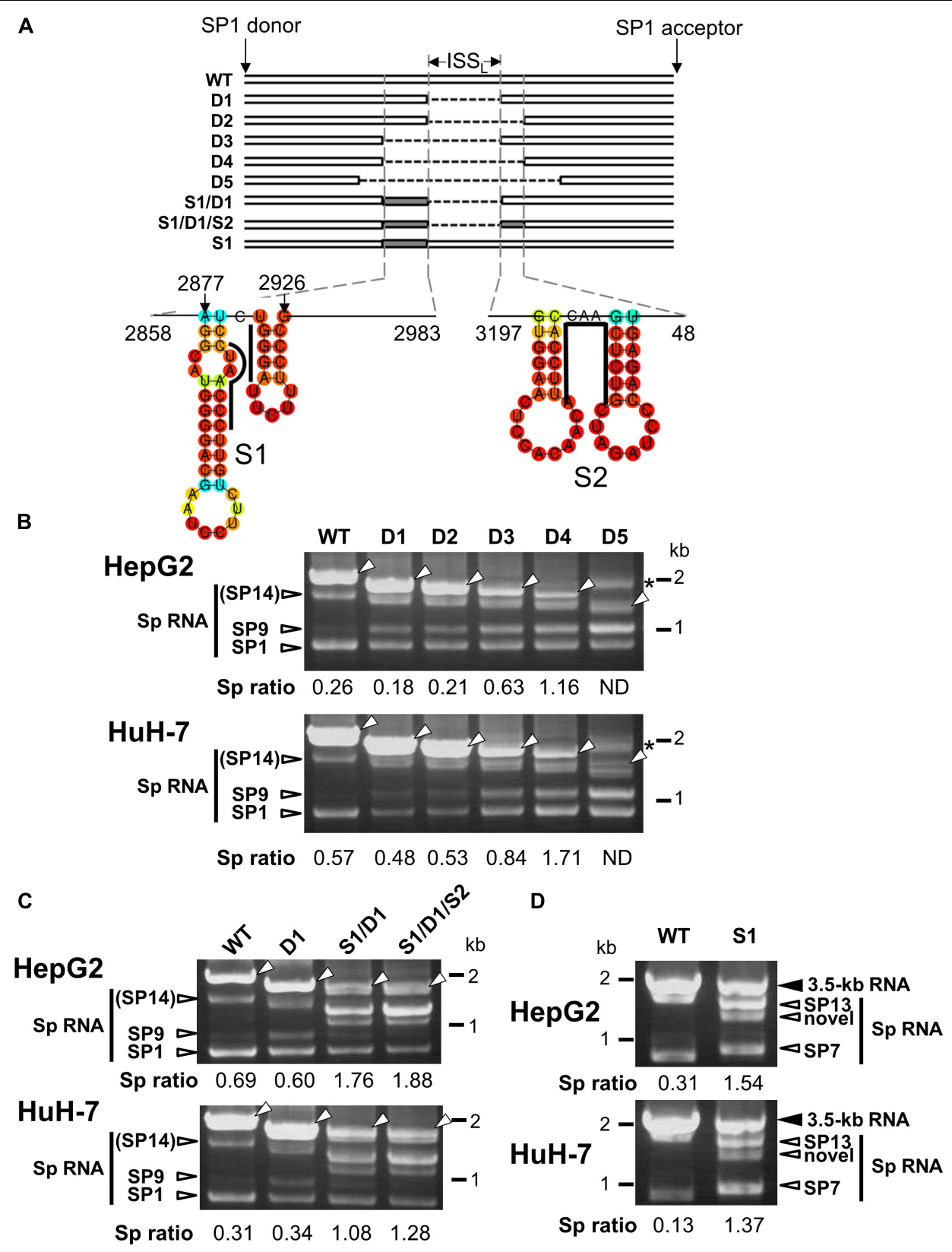

FIGURE 3 | Characterization of the intronic sequence important for silencing of the $3.5 \mathrm{~kb}$ RNA splicing. (A) A schematic representation of mutated HBV genomes derived from GT-C with deletions and/or substitutions within the major intron region is shown. Putative secondary structures predicted by the CentroidFold (http://rtools.cbrc.jp/centroidfold/) in nt 2858-2983 and 3197-48 regions are indicated. Each predicted base pair is colored with the heat color gradation from blue to red corresponding to the base-pairing probability. Bold lines are drawn along the sequences targeting to introduce substitution mutations S1 and S2. (B) RT-PCR analysis of HBV RNAs expressed from WT and a series of deletion-mutated HBV genomes (D1-D5) in HepG2 and HuH-7 cells. The quantity ratio of the spliced RNAs to the unspliced RNA (Sp ratio) was determined. ND, not determined. Open arrows, unspliced forms. *cDNA products with aberrant sizes. (C) As described in (B) but HBV mutated genomes S1/D1 and S1/D1/S2 in addition to WT and D1 were used for RNA expression. (D) As in (C) but S1 mutant and WT were expressed in the cells. Bands corresponding to unspliced $3.5 \mathrm{~kb}$ RNA and its spliced forms (Sp RNA) were indicated.

(Chowdhury et al., 2011). Since we also found that the HBV major intron region contains a cis-regulatory element that silences the usage of adjacent splice sites of the $3.5 \mathrm{~kb}$ RNA in the context with the viral genome replication as shown above, a series of the 1.24-fold HBV genome constructs derived from GT-C with partial deletions corresponding to the intronic sequence were generated to identify the region that contributes to negative regulation of the splicing (Figure 3A). Their splicing silencer activity was analyzed by comparing processing patterns of expressed RNAs in HepG2 and $\mathrm{HuH}-7$ cells (Figure 3B). Either deletion mutant D1 or D2, in which ISS $_{\mathrm{L}}$ or nt 2984-48 region was deleted, respectively, has a moderate influence on 


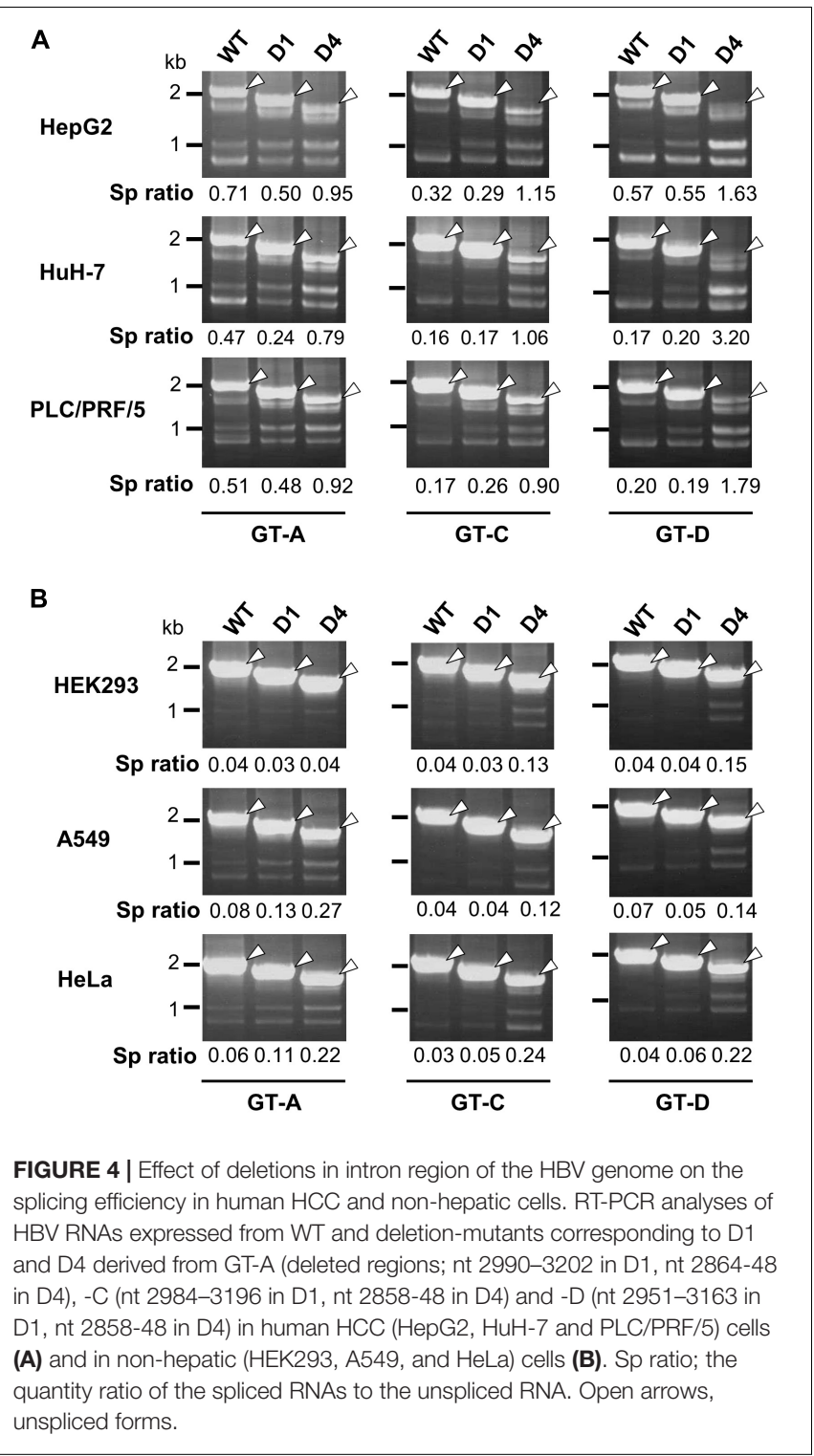

the ratio of spliced/unspliced RNAs. Expression of D3 mutant resulted in a marked increase in the ratio and D4 displayed the highest splicing efficiency. Further deletion(s) such as D5 caused generation of some aberrant RNA product(s) whose size(s) are larger than the expected unspliced form.

The possible contribution of the regions spanning nt 28582983 and 3197-48 which potentially contain several stem-loop structures (Figure 3A) to the splicing regulation was further tested by introducing substitution mutations in the respective sequences of D1 mutant to disrupt the base-pairs of putative stem-loops, leading to changing the secondary structures of the region. Expression of both mutants; S1/D1 and S1/D1/S2 resulted in generation of novel spliced RNA species and increasing significantly the ratio of spliced/unspliced RNAs (Figure 3C). To pinpoint the sequence response for the novel intronic splicing silencer, a mutated genome S1 with substitutions at nt 29022916 region (Figure 3A) was assessed. Expression of S1 led to a decreased level of unspliced RNA and change in the spliced RNA pattern (Figure 3D), indicating reduction of the splicing silencer activity by the substitution mutation. Nucleotide sequencing showed that previously reported spliced RNAs; SP7 and SP13 (Bayliss et al., 2013) and a novel spliced RNA (Supplementary Figure S4) were detectable in cells expressing S1 mutant genome.

In nt 2858-2983 region, two stem-loop structures that are important for the silencing activity are predicted to exist in the sequence spanning nt 2877-2926 (Figure 3A). Collectively, we concluded that the intron region of nt 2877-2926, located upstream from the previously reported ISS $_{\mathrm{L}}$, functions as a potent splicing silencer and is termed as ISS $S_{U L}$ (ISS upstream from $\mathrm{ISS}_{\mathrm{L}}$ ). Further experiments with the mutated genome D4 showed that higher splicing ratios in cells expressing D4 compared to expression of D1 and wild-type genomes were observed in any cell lines as well as HBV genotypes tested. The results suggested that the sequence containing ISS $_{U L}$ and ISS $_{\mathrm{L}}$ in the major intron region of $3.5 \mathrm{~kb}$ RNA displayed the splicing silencing activity independent of HBV GTs and of cell backgrounds (Figures 4A,B).

\section{Characterization of Genotype/Strain-Dependent Variation in the Efficiency of the 3.5-kb RNA Splicing}

As a characterization of splicing regulation of HBV RNAs, we next asked how splicing efficiency of $3.5 \mathrm{~kb}$ RNA varies among $\mathrm{HBV}$ genotypes or strains. Four kinds of HBV genomes derived from GT-A, -B, -C, and -D were expressed in HepG2 and HuH-7 cells and the ratios of spliced/unspliced RNAs were compared at 2 days post-transfection (Figure 5A). Among HBV GTs/strains tested, the splicing efficiency of the $3.5 \mathrm{~kb}$ RNA expressed from the GT-A clone and that from the GT-C clone was highest and lowest, respectively, in both cells. Similar results were obtained when the splicing efficiency was determined at 4 days posttransfection (Supplementary Figure S5). To identify the viral sequence that has potentially influence on GT/strain-dependent variation in the splicing efficiency, parts of $\mathrm{HBV}$ sequence from the GT-C clone were serially replaced by the corresponding parts from the GT-A clone in the genome (Figure 5B) and expressed. The $3.5 \mathrm{~kb}$ RNA expressed from the chimeric clone Ce/Ae2 that carries GT-A-derived nt 2605-248 sequence in the GT-C basis was efficiently spliced comparable to that from the wild-type GT-A clone (Figure 5C). The $3.5 \mathrm{~kb}$-RNA splicing from chimeric mutants $\mathrm{Ce} / \mathrm{Ae} 5-\mathrm{Ce} / \mathrm{Ae} 7$ was further assessed. Splicing efficiency of the viral RNAs derived from Ce/Ae5 and $\mathrm{Ce} / \mathrm{Ae} 6$ but not from Ce/Ae7 was increased compared to that from wild-type GT-C clone (Figure 5D). For the regionswapping experiment, a chimeric clone Ae/Ce that is GT-A based counterparts of $\mathrm{Ce} / \mathrm{Ae} 2$ was constructed (Supplementary Figure S6A). As expected, the $3.5 \mathrm{~kb}$ RNA expressed from Ae/Ce were less efficiently spliced compared to the wild-type GT-A clone and the splicing efficiency of Ae/Ce-derived RNA was as low as that of the wild-type GT-C clone (Supplementary Figure S6B). Although evidence obtained from the GT-A and -C clones in this study was limited, it may be likely that a considerable part (795 nt) within the major intron region is possibly involved 
A
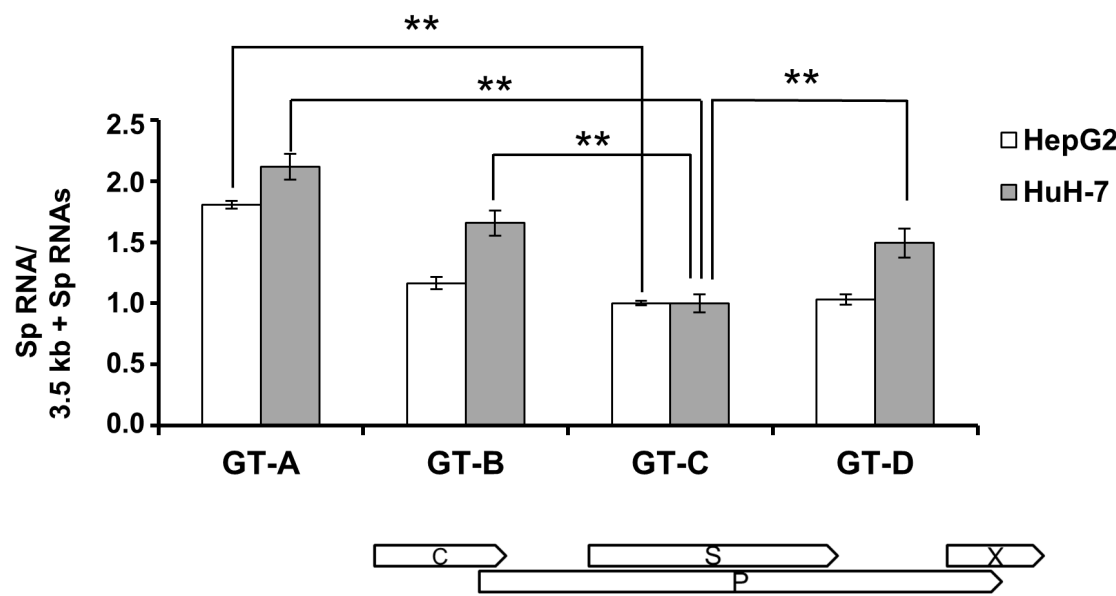

B

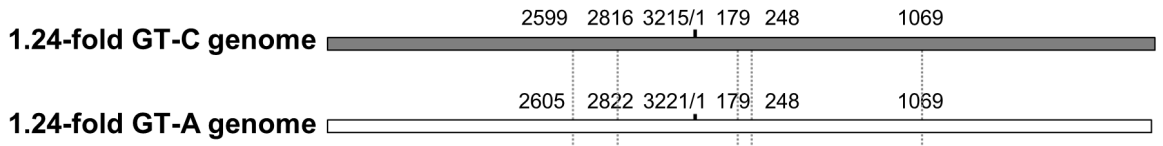

HBV Ce/Ae 1

HBV CelAe 2

HBV Ce/Ae 3

HBV Ce/Ae 4

HBV CelAe 5

HBV CelAe 6 HBV Ce/Ae 7

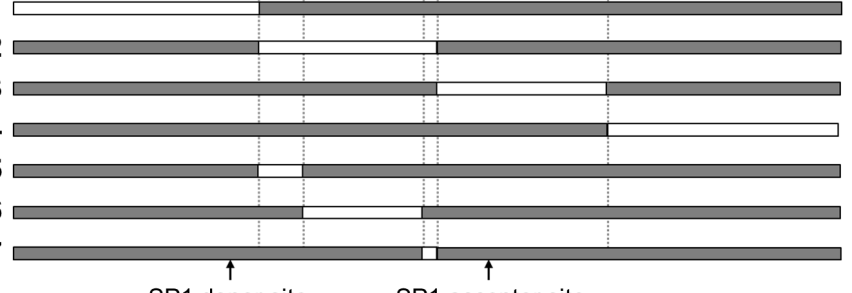

SP1 donor site SP1 acceptor site

C
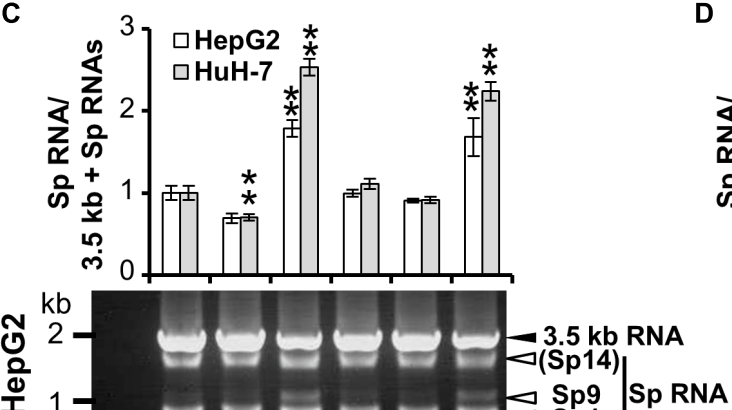

오

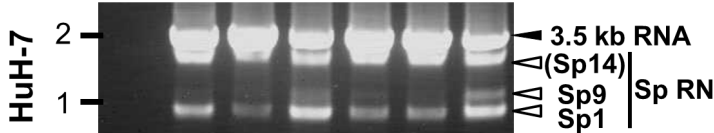

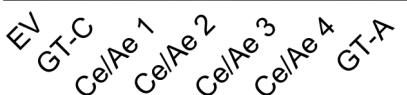

D

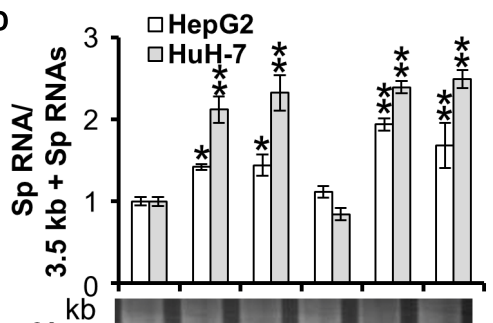

๙ $^{\mathrm{kb}}-3.5 \mathrm{~kb}$ RNA

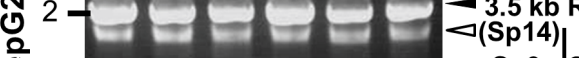

$\checkmark$ Sp9 $\operatorname{Sp~RNA}$

Sp1

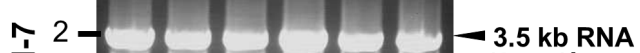

곤

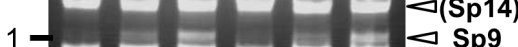

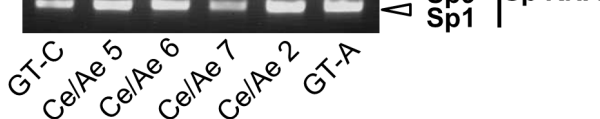

FIGURE 5 | Diversity in the splicing efficiency among HBV genotypes/strains. (A) pUC-HB-Ae (GT-A), -Bj56 (GT-B), -Ce (GT-C) or -D Ind60 (GT-D) was transfected into HepG2 and HuH-7 cells. Two days after the transfection, total RNA was extracted from cells and analyzed by qRT-PCR to determine the levels of $3.5 \mathrm{~kb}$ RNA and spliced RNAs. The quantity ratios of the spliced RNAs to total $3.5 \mathrm{~kb}$ RNA derived RNA species were calculated and that of GT-C was set to 1 . (B) A schematic representation of a series of mutated HBV genomes replacing parts of the GT-C sequence with corresponding parts of GT-A sequence. Positions of donor and acceptor sites of SP1 RNA are indicated. (C) qRT-PCR analysis was performed to determine $3.5 \mathrm{~kb}$ RNA and spliced RNA levels in HepG2 and HuH-7 cells transfected with pUC-HB- Ce (GT-C), -Ae (GT-A), HBV Ce/Ae1, /Ae2, /Ae3 or /Ae4. The quantity ratios of the spliced RNAs to total $3.5 \mathrm{~kb}$ RNA derived RNA species were calculated and those in GT-C-expressing cells were set to 1 (upper). Representative pattern for RT-PCR result indicating expression of unspliced and spliced (Sp) RNAs derived from $3.5 \mathrm{~kb}$ RNA in the transfected HepG2 (middle) and HuH-7 cells (lower). (D) As in (C) but mutated genomes HBV Ce/Ae5, /Ae6, /Ae7 as well as GT-C, $-\mathrm{A}$ and HBV Ce/Ae2 were used for expression of HBV RNAs. Values represent mean \pm SD (HepG2; $n=2, \mathrm{HuH}-7 ; n=3) .{ }^{*} p<0.05,{ }^{* *} p<0.01$, by one-way ANOVA followed by Dunnett's test compared to GT-C. 
in GT/strain-dependent variation in the efficiency of the $3.5-\mathrm{kb}$ RNA splicing.

\section{DISCUSSION}

It is generally considered that alternative RNA splicing is essential for the life cycles of nuclear-replicating viruses because their post-transcriptional processing is one of the major mechanisms in increasing the diversity of proteins translated from a limited number of viral genes required to initiate and maintain the viral proliferation. However, knowledge of a cell-type dependent manner of the viral RNA splicing and its regulatory mechanism is quite limited (Ge and Manley, 1990; Butera et al., 1994). In this study, through analysis with 13 kinds of cell lines, we found cell-type dependency of splicing of HBV $3.5 \mathrm{~kb}$ RNA. The viral RNA is relatively efficiently spliced in human HCC cells but not in cells derived from human hepatic stellate, mouse hepatoma and human non-hepatic cells (Figure 1C). It is possible that difference in transcription efficiency of the HBV genome may have an influence on the splicing regulation. In fact, expression levels of HBV RNAs in human HCC-derived, HepG2 and HuH-7 cells were highest among cell lines tested. However, the HBV RNA levels expressed in other human HCC-derived, PLC/PRF/5, FLC7 and ORL8c cells where the viral RNAs can be efficiently spliced like HepG2 and HuH-7 cells were quite low. The expression levels in PLC/PRF/5, FLC7 and ORL8c cells were lower compared to those in some non-HCC cells such as HEK293 and HEK293T cells. Thus, it is likely that cell-type dependency of HBV splicing efficiency cannot be explained only by expression level of the viral RNAs. These findings may suggest that RNA splicing is a key determinant for optimizing regulation of HBV life cycle and the host range. HBV RNA splicing is a common event during chronic infection (Sommer and Heise, 2008). Several singly and multiply spliced isoforms derived from the HBV $3.5 \mathrm{~kb}$ RNA have been identified in liver tissues and sera obtained from HBV-infected individuals as well as tissues and cultured cells of HBV-replicating models such as transgenic mice and transfected cells (Sommer and Heise, 2008). Although the spliced variants derived from $3.5 \mathrm{~kb}$ RNA are basically unable to replicate autonomously because of no production of functional polymerase, it may be possible to replicate in the presence of unspliced RNA intermediate, the pregenomic RNA. The most frequently detectable HBV spliced form SP1, which is generated through the removal of a $\sim 1.2 \mathrm{~kb}$ intron from the 3.5 kb RNA (Su et al., 1989; Suzuki et al., 1989). SP1 was reported to encode an hepatitis B splice-generated protein HBSP, which contains a small portion of the $\mathrm{N}$-terminal viral polymerase fused with a new open-reading frame produced by the splicing (Soussan et al., 2000). In addition, SP1 has the potential to encode $\mathrm{HBcAg}$ with the C-terminal cysteine deleted. A doubly spliced form, SP10, has also been characterized and reported to encode $\sim 15 \mathrm{kDa}$ hepatitis B doubly spliced protein (Chen et al., 1989). Although possible implication of these proteins encoded by spliced RNAs in HBV replication has been suggested (Ma et al., 2009; Bayliss et al., 2013), further studies are required to explore and evaluate the biological significance of the spliced
RNAs and proteins encoded by the RNAs in HBV replicative life cycle.

In general, sequence features/elements outside of the canonical splice site and branch site sequence located near the $5^{\prime}$ and $3^{\prime}$ ends of introns can be involved in recognizing authentic splice sites by the splicing machinery among a lot of sequences that match the consensus motifs. These elements are conventionally classified as exonic splicing enhancers, exonic splicing silencers, intronic splicing enhancers and ISSs. As cis-regulatory elements for splicing of HBV $3.5 \mathrm{~kb}$ RNA, the PRE located at nt 1217-1582 (Heise et al., 2006) and the ISS $_{\mathrm{L}}$ located at 2951-3163 (nucleotide numbering according to AY945307) (Chowdhury et al., 2011) were identified using constructs under the control by the heterologous promoters. In this study, given the results showing the difference in cell-type dependency of the splicing efficiency between HBV and SV40 (Figure 1C and Supplementary Figure S2), intron-swapping experiments were performed (Figure 2). The results suggest that the certain sequence(s) within the exon region of majority of the HBV spliced RNAs positively regulate the splicing in the cell-type dependent manner. From further analyses with deletion and substitution mutations within the major HBV intron region, we identified a novel element ISS $\mathrm{UL}$ that functions as ISS located at nt 2877-2926 region, upstream of ISS $_{\mathrm{L}}$. It appears that the splicing silencing activity of ISS $\mathrm{UL}_{\mathrm{L}}$ is more potent compared to that of $\operatorname{ISS}_{\mathrm{L}}$ (Figures 3C,D) and that the intron region containing ISS $_{\mathrm{UL}}$ and ISS $\mathrm{I}_{\mathrm{L}}$ functions effectively independent of the viral GTs/strains and of cell backgrounds (Figure 4). By comparing data between hepatic (Figure 4A) and non-hepatic (Figure 4B) cells, an increase in the ratio of spliced to unspliced products by deletion with nt 2864-48 region in non-hepatic cells seems to be less evident than that in hepatic cells. One reason may be that, in addition to ISS $S_{\mathrm{UL}}$ and ISS $\mathrm{L}_{\mathrm{L}}$, a putative positive regulator in the exon region, as mentioned above, is potentially involved in controlling the HBV splicing particularly in hepatic cells. The significance of RNA secondary structures in molecular mechanisms including alternative splicing is well described. It is thus important to consider that structural conformation is a key to the element for splicing regulation. Interestingly, the ISS $\mathrm{UL}_{\mathrm{L}}$ is predicted to contain two stem-loop structures in close proximity (Figure 3A). Database analyses, together with the secondary structure prediction, demonstrated that nucleotide sequence in the ISS $\mathrm{UL}_{\mathrm{L}}$ region is largely well conserved and in particular the second stem-loop structure is completely conserved among GT-A-D clones.

It is known that most of all corresponding donor and acceptor sites for major splicing derived from the $3.5 \mathrm{~kb}$ RNA are correctly identified in consensus sequences of the viral GTs A-D (Candotti and Allain, 2017). In contrast, several studies with clinical samples reported that efficiency of the $3.5 \mathrm{~kb}$ RNA splicing and nature of splice variants differ from patient to patient ranging from undetectable splicing to extensive and genotype/viral straindependent splicing (Wu et al., 1991; Gunther et al., 1997; Sommer et al., 2000; El Chaar et al., 2012).

In this study, the ratio of spliced to unspliced $3.5 \mathrm{~kb}$ RNA expressed from four kinds of HBV clones individually derived from GTs A-D in HepG2 and HuH-7 cells was compared. 
The ratios obtained from GT-A and GT-C clones tended to be highest and lowest, respectively. Further findings from the region-swapping experiments suggest that $\sim$ two-third part of the major intron region may be participated in genotype/strain-dependent variation in the splicing efficiency. Thus, besides the identified ISS regions that are well conserved among HBV strains, unidentified cis-elements that are responsible for positive- or negative splicing regulation and are functional in a GT/strain-dependent fashion may be present within the intron.

Upon infection, HBV hijacks the host splicing machinery to develop regulatory mechanisms for alternative splicing of the viral transcripts. Our finding that the $\mathrm{HBV} 3.5 \mathrm{~kb}$ RNA is efficiently spliced in human hepatic cells among various types of cells may suggest its biological significance as a host range determinant. In contrast, maintenance of an appropriate amount of the unspliced form is indispensable to supplying the replication intermediate during the viral replication cycles. Thus, a crucial aspect for HBV alternative splicing is accurate control of proper ratios of spliced to unspliced forms. A shift in the viral splicing and change in the spliced/unspliced RNA ratio might be achieved by altering the balance of nuclear factors that recognize either splicing enhancers or silencers and mediate the catalytic steps of the splicing reaction. Further studies on mapping putative exonic enhancer(s) responsible for acting in a cell-type dependent manner, identification of host factors that positively and negatively regulate the splicing and elucidation of their regulatory mechanisms are important to better understand $\mathrm{HBV}$ RNA regulation and the significance of the viral alternative splicing.

\section{REFERENCES}

Bayliss, J., Lim, L., Thompson, A. J., Desmond, P., Angus, P., Locarnini, S., et al. (2013). Hepatitis B virus splicing is enhanced prior to development of hepatocellular carcinoma. J. Hepatol. 59, 1022-1028. doi: 10.1016/j.jhep.2013. 06.018

Butera, S. T., Roberts, B. D., Lam, L., Hodge, T., and Folks, T. M. (1994). Human immunodeficiency virus type 1 RNA expression by four chronically infected cell lines indicates multiple mechanisms of latency. J. Virol. 68, 2726-2730.

Candotti, D., and Allain, J.-P. (2017). Biological and clinical significance of hepatitis B virus RNA splicing: an update. Ann. Blood 2, 1-14.

Chen, P. J., Chen, C. R., Sung, J. L., and Chen, D. S. (1989). Identification of a doubly spliced viral transcript joining the separated domains for putative protease and reverse transcriptase of hepatitis B virus. J. Virol. 63, 4165-4171.

Chida, T., Ito, M., Nakashima, K., Kanegae, Y., Aoshima, T., Takabayashi, S., et al. (2017). Critical role of CREBH-mediated induction of transforming growth factor beta2 by hepatitis $\mathrm{C}$ virus infection in fibrogenic responses in hepatic stellate cells. Hepatology 66, 1430-1443. doi: 10.1002/hep.29319

Chowdhury, J. B., Roy, D., and Ghosh, S. (2011). Identification of a unique splicing regulatory cluster in hepatitis B virus pregenomic RNA. FEBS Lett. 585, 3348-3353. doi: 10.1016/j.febslet.2011.09.026

Duriez, M., Mandouri, Y., Lekbaby, B., Wang, H., Schnuriger, A., Redelsperger, F., et al. (2017). Alternative splicing of hepatitis B virus: a novel virus/host interaction altering liver immunity. J. Hepatol. 67, 687-699. doi: 10.1016/j.jhep. 2017.05.025

El Chaar, M., El Jisr, T., and Allain, J. P. (2012). Hepatitis B virus DNA splicing in lebanese blood donors and genotype A to E strains: implications for hepatitis B virus DNA quantification and infectivity. J. Clin. Microbiol. 50, 3159-3167. doi: 10.1128/JCM.01251- 12

\section{AUTHOR CONTRIBUTIONS}

NI, KN, SS, and MI performed the experimental work. NI and KN prepared the figures and table. KN and TS contributed in study conception and data interpretation. NI, KN, and TS wrote the manuscript. All authors participated in data interpretation and contributed to manuscript revision.

\section{FUNDING}

This work was supported by the Japan Agency for Medical Research and Development, AMED (JP17fk0310119h0001, JP18fk0310103h0802, JP17fk0310104h0001, JP17fk0310102h0001, JP18fk0210009h0903, JP18fk0210022h0702, and JP18fk0210040h 0001) and from the Ministry of Education, Culture, Sports, Science, and Technology, Japan.

\section{ACKNOWLEDGMENTS}

We thank Masashi Mizokami for providing plasmids. We are also grateful to T. Mochizuki for secretarial work and to S. Nomura and M. Yamamoto for their technical assistance.

\section{SUPPLEMENTARY MATERIAL}

The Supplementary Material for this article can be found online at: https://www.frontiersin.org/articles/10.3389/fmicb. 2019.00207/full\#supplementary-material

El-Serag, H. B. (2012). Epidemiology of viral hepatitis and hepatocellular carcinoma. Gastroenterology 142, 1264-1273.e1. doi: 10.1053/j.gastro.2011. 12.061

Ge, H., and Manley, J. L. (1990). A protein factor, ASF, controls cell-specific alternative splicing of SV40 early pre-mRNA in vitro. Cell 62, 25-34. doi: 10.1016/0092-8674(90)90236-8

Gunther, S., Sommer, G., Iwanska, A., and Will, H. (1997). Heterogeneity and common features of defective hepatitis B virus genomes derived from spliced pregenomic RNA. Virology 238, 363-371. doi: 10.1006/viro.1997.8863

Heise, T., Sommer, G., Reumann, K., Meyer, I., Will, H., and Schaal, H. (2006). The hepatitis B virus PRE contains a splicing regulatory element. Nucleic Acids Res. 34, 353-363. doi: 10.1093/nar/gkj440

Homma, S., Nagamori, S., Fujise, K., Hasumura, S., Sujino, H., Matsuura, T., et al. (1990). Establishment and characterization of a human hepatocellular carcinoma cell line JHH-7 producing alpha -fetoprotein and carcinoembryonic antigen-changes in secretion of AFP and CEA from JHH-7 cells after heat treatment. Hum. Cell 3, 152-157.

Huang, C., Xie, M. H., Liu, W., Yang, B., Yang, F., Huang, J., et al. (2011). A structured RNA in hepatitis B virus post-transcriptional regulatory element represses alternative splicing in a sequence-independent and positiondependent manner. FEBS J. 278, 1533-1546. doi: 10.1111/j.1742-4658.2011. 08077.x

Kato, N., Mori, K., Abe, K., Dansako, H., Kuroki, M., Ariumi, Y., et al. (2009). Efficient replication systems for hepatitis $\mathrm{C}$ virus using a new human hepatoma cell line. Virus Res. 146, 41-50. doi: 10.1016/j.virusres.2009.08.006

Li, Y., Ito, M., Sun, S., Chida, T., Nakashima, K., and Suzuki, T. (2016). LUC7L3/CROP inhibits replication of hepatitis B virus via suppressing enhancer II/basal core promoter activity. Sci. Rep. 6:36741. doi: 10.1038/ srep36741 
Ma, Z. M., Lin, X., Wang, Y. X., Tian, X. C., Xie, Y. H., and Wen, Y. M. (2009). A double-spliced defective hepatitis B virus genome derived from hepatocellular carcinoma tissue enhanced replication of full-length virus. J. Med. Virol. 81, 230-237. doi: 10.1002/jmv.21393

Nakanishi, A., Chapellier, B., Maekawa, N., Hiramoto, M., Kuge, T., Takahashi, R. U., et al. (2008). SV40 vectors carrying minimal sequence of viral origin with exchangeable capsids. Virology 379, 110-117. doi: 10.1016/j.virol.2008.06.032

Redelsperger, F., Lekbaby, B., Mandouri, Y., Giang, E., Duriez, M., Desire, N., et al. (2012). Production of hepatitis B defective particles is dependent on liver status. Virology 431, 21-28. doi: 10.1016/j.virol.2012.05.008

Rosmorduc, O., Petit, M. A., Pol, S., Capel, F., Bortolotti, F., Berthelot, P., et al. (1995). In vivo and in vitro expression of defective hepatitis B virus particles generated by spliced hepatitis B virus RNA. Hepatology 22, 10-19.

Schneider, C. A., Rasband, W. S., and Eliceiri, K. W. (2012). NIH image to imagej: 25 years of image analysis. Nat. Methods 9, 671-675. doi: 10.1038/nmeth.2089

Shibata, N., Watanabe, T., Okitsu, T., Sakaguchi, M., Takesue, M., Kunieda, T., et al. (2003). Establishment of an immortalized human hepatic stellate cell line to develop antifibrotic therapies. Cell Transplant. 12, 499-507. doi: 10.3727/ 000000003108747064

Sommer, G., and Heise, T. (2008). Posttranscriptional control of HBV gene expression. Front. Biosci. 13:5533-5547. doi: 10.2741/3097

Sommer, G., Van Bommel, F., and Will, H. (2000). Genotype-specific synthesis and secretion of spliced hepatitis B virus genomes in hepatoma cells. Virology 271, 371-381. doi: 10.1006/viro.2000.0331

Soussan, P., Garreau, F., Zylberberg, H., Ferray, C., Brechot, C., and Kremsdorf, D. (2000). In vivo expression of a new hepatitis B virus protein encoded by a spliced RNA. J. Clin. Invest. 105, 55-60. doi: 10.1172/JCI8098

Soussan, P., Pol, J., Garreau, F., Schneider, V., Le Pendeven, C., Nalpas, B., et al. (2008). Expression of defective hepatitis B virus particles derived from singly spliced RNA is related to liver disease. J. Infect. Dis. 198, 218-225. doi: 10.1086/ 589623

Su, T. S., Lai, C. J., Huang, J. L., Lin, L. H., Yauk, Y. K., Chang, C. M., et al. (1989). Hepatitis B virus transcript produced by RNA splicing. J. Virol. 63, 4011-4018.
Sugauchi, F., Kumada, H., Acharya, S. A., Shrestha, S. M., Gamutan, M. T., Khan, M., et al. (2004a). Epidemiological and sequence differences between two subtypes (Ae and Aa) of hepatitis B virus genotype A. J. Gen. Virol. 85, 811-820.

Sugauchi, F., Kumada, H., Sakugawa, H., Komatsu, M., Niitsuma, H., Watanabe, H., et al. (2004b). Two subtypes of genotype B (Ba and Bj) of hepatitis B virus in Japan. Clin. Infect. Dis. 38, 1222-1228.

Sun, S., Nakashima, K., Ito, M., Li, Y., Chida, T., Takahashi, H., et al. (2017). Involvement of PUF60 in transcriptional and post-transcriptional regulation of hepatitis B virus pregenomic RNA expression. Sci. Rep. 7:12874. doi: 10.1038/ s41598-017-12497-y

Suzuki, T., Masui, N., Kajino, K., Saito, I., and Miyamura, T. (1989). Detection and mapping of spliced RNA from a human hepatoma cell line transfected with the hepatitis B virus genome. Proc. Natl. Acad. Sci. U.S.A. 86, 8422-8426. doi: $10.1073 /$ pnas.86.21.8422

Terre, S., Petit, M. A., and Brechot, C. (1991). Defective hepatitis B virus particles are generated by packaging and reverse transcription of spliced viral RNAs in vivo. J. Virol. 65, 5539-5543.

Wu, H. L., Chen, P. J., Tu, S. J., Lin, M. H., Lai, M. Y., and Chen, D. S. (1991). Characterization and genetic analysis of alternatively spliced transcripts of hepatitis B virus in infected human liver tissues and transfected HepG2 cells. J. Virol. 65, 1680-1686.

Conflict of Interest Statement: The authors declare that the research was conducted in the absence of any commercial or financial relationships that could be construed as a potential conflict of interest.

Copyright (c) 2019 Ito, Nakashima, Sun, Ito and Suzuki. This is an open-access article distributed under the terms of the Creative Commons Attribution License (CC BY). The use, distribution or reproduction in other forums is permitted, provided the original author(s) and the copyright owner(s) are credited and that the original publication in this journal is cited, in accordance with accepted academic practice. No use, distribution or reproduction is permitted which does not comply with these terms. 